\title{
Sensor for Tongue-pressure Measurement Based on Extreme Learning Machine
}

\author{
Chen Yifei, Xia Rongfei, and Feng Yongjian* \\ School of Aerospace Engineering, Xiamen University, 422 Siming South Road, Xiamen 361005, China
}

(Received April 18, 2018; accepted July 27, 2018)

Keywords: tongue pressure, sensor, extreme learning machine

Dysphagia is a common complication of cerebrovascular accidents, and greatly affects the prognosis of patients. To meet the portability requirement for instruments used in the diagnosis of dysphagia in clinical settings, a novel sensor for measuring tongue pressure is designed to help doctors evaluate the severity of dysphagia. A sensor with a special beam structure converts the deformation of strain gauges to analog voltage signals through a Wheatstone bridge, then translates the result to a value for tongue pressure. The relationship between the tongue pressure and voltage is fitted by a least squares method and also by using an extreme learning machine. The two sets of results are compared and the superiority of using the extreme learning machine is verified. For the designed tongue-pressure sensor with an outer diameter of $10 \mathrm{~mm}$ and a thickness of $3 \mathrm{~mm}$, the detection range is from 0 to $15 \mathrm{kPa}$, and the relative error of measurement can be controlled within $0.91 \%$.

\section{Introduction}

With the advance of the aging problem, cerebrovascular diseases have become a severe threat to human health. According to surveys, dysphagia is the common complication of these diseases, and its incidence reaches $22-65 \% .^{(1,2)}$ Dysphagia can also lead to severe medical complications, including dehydration, malnourishment, pressure ulcer, pneumonia, and cognitive disorders. This will reduce the life quality of patients, endanger patients' physical and mental health, and also give rise to heavy economic burden to families, society, and government.

Swallowing physiologically includes five stages: cognitive, oral, preparation, pharyngeal, and esophageal. ${ }^{(3)}$ In these processes, the tongue has important roles: assisting in mastication, gustatory sensation, inducing salivation, bolus formation, and propelling the bolus into the pharynx. ${ }^{(4)}$ Hence the accurate measurement of tongue pressure is important in treating dysphagia. For now, the main methods of checking for dysphagia in clinical settings are videofluorography and swallowing endoscopy. These methods display swallowing dynamically from the side, front, and back vividly with images. However, videofluorography involves radiation and swallowing endoscopy has operating difficulties. Therefore, these two methods

*Corresponding author: e-mail: yjfeng@xmu.edu.cn https://doi.org/10.18494/SAM.2018.1966 
are difficult to use widely and repeatedly. Through an extensive survey of foreign research on the detection of tongue pressure, we found that various sensors have been applied to tonguepressure measurement in recent years. ${ }^{(5-12)}$ However, most of the research was based on an experimental model. Although the measurement values are accurate, equipment installation is complex, and hence unsuitable for clinical applications. A novel sensor just $0.1 \mathrm{~mm}$ thick is applied to tongue-pressure measurement, and is, by far, the smallest sensor currently available. The main feature of this sensor is that strain gauges are fastened directly to the mouth, which leads to many problems such as difficulty in attachment and in guaranteeing measurement precision.

We design a microsensor with a beam structure for measuring tongue pressure that is based on the extreme learning machine. This sensor is based on the piezoresistive principle and converts deformation of strain gauges to analog voltage signals through a Wheatstone bridge. For the problem of nonlinearity between the sensor input and output, we adopted a least squares method and the extreme learning machine to realize nonlinear compensation. The measurement range of the designed tongue-pressure sensor is from 0 to $15 \mathrm{kPa}$, and the relative error of measurement can be controlled within $0.91 \%$.

\section{Sensor Design}

\subsection{Structure design of sensor}

\subsubsection{Operation principle}

The piezoresistive effect is a physical phenomenon produced by the change in the resistance of a metal conductor upon its deformation such as stretching or shortening. ${ }^{(13)}$ For a stripe conductor with length 1 , cross-sectional area $S$, and electrical resistivity $\rho$, its resistance is

$$
R=\rho \frac{1}{s}
$$

Metal conductors also become deformed upon the application of an external force, which also changes the resistance. Take the log of Eq. (1) and then take its derivative

$$
\frac{d R}{R}=\frac{d L}{L}-\frac{d S}{S}+\frac{d \rho}{\rho}
$$

$d L / L=\varepsilon$ denotes the axial strain and $d S / S=2(d D / D)$, where $D$ is the cross-sectional diameter of metallic conductors. On the basis of the theory of the mechanics of materials, when metal wire is subjected to a radial force,

$$
\frac{d D}{D}=-\mu \frac{d L}{L}
$$


where $\mu$ is Poisson's ratio. Substituting Eq. (3) into Eq. (2) gives

$$
\frac{d R}{R}=\frac{d \rho}{\rho}+(1+2 \mu) \varepsilon
$$

$d \rho / \rho$ is the relative change in the resistivity, the ratio $d \rho / \rho$ relative to the longitudinal stress of the semiconductor materials is constant, that is, $d \rho / \rho=\pi E \varepsilon . \pi$ is the piezoresistive coefficient of the semiconductor. By elementary operation, we obtain

$$
\frac{d R}{R}=(1+2 \mu+\pi E) \varepsilon
$$

$1+2 \mu$ represents the geometrical deformation of materials. $\pi E$ is the piezoresistive effect and changes with resistivity. $E$ is Young's modulus.

The results of the experiment indicated that $1+2 \mu$ in Eq. (2) is negligible when the piezoresistive effect $\pi E$ is much larger than the relative change in the resistivity caused by material deformation. Therefore,

$$
\frac{d R}{R} \approx \pi E \varepsilon,
$$

which is the relationship between the change in resistivity and stress.

\subsubsection{Structure design}

The tongue pressure on the maxilla differs depending on the state of the tongue. At rest, the tongue pressure is the lowest, usually $2-3 \mathrm{kPa}$. During swallowing, the tongue pressure is the greatest and can reach $20-30 \mathrm{kPa}$. Thus, we need a sensor with a small measurement range, but with high sensitivity. Moreover, the sensor needs to be deployed on the central axis of the maxilla and buried in the denture. Therefore, the sensor size has a certain limit. The basic parameters of sensors are as below.

Effective measuring range: $0-4.5 \mathrm{~N}$;

Dimensions: within $15 \times 15 \mathrm{~mm}^{2}$.

Because of the above requirements, the sensor structure is designed to be circular with an external diameter of $12 \mathrm{~mm}$. The basic structure of the sensor is shown in Fig. 1. It mainly comprises six components: retraining washer, beam, support frame, metal pad, silicone pad, and metal shell. The beam is deformed when pressure is exerted on the resin base plate, as detected by the strain gauge, enabling pressure measurement.

The core of the sensor has a beam structure, with the shape shown in Fig. 2. There are two resistance strain gauges, R1 and R2, attached to the middle arm of the beam, and they deform with beam deformation under pressure. The resistances of R1 and R2 also change. The change in resistance is converted to an analog voltage signal using a Wheatstone bridge. Then, the 


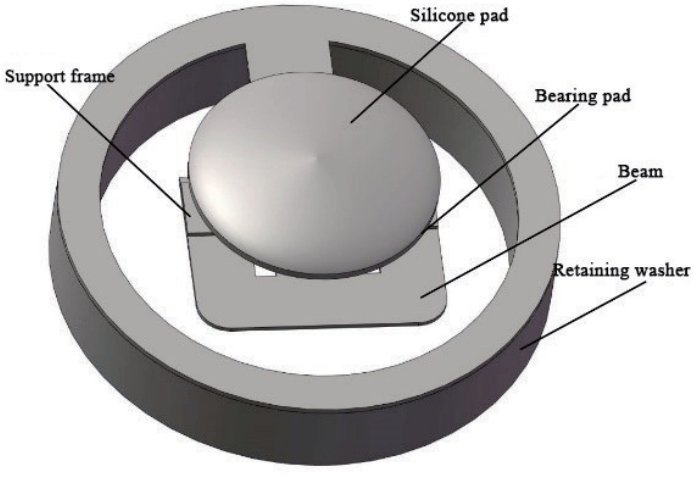

Fig. 1. Sensor structure.

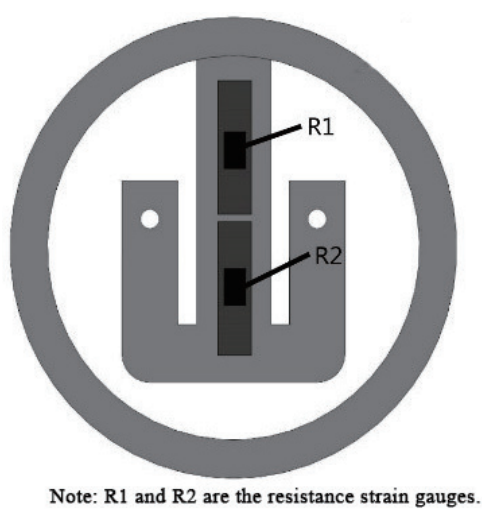

Fig. 2. Tablet beam structure.

value of pressure is obtained by converting the voltage signal. The merit of this method is that there are two resistance strain gauges, R1 and R2, attached to the middle arm of the beam, under the action of a force, R1 stretches and R 2 contracts. The proposed structure can result in large resistance changes with small deformation. Owing to the weak output signals of the strain gauges, they can also be used to reduce amplification distortion.

Figure 3 shows the sensor with an outside diameter of $10 \mathrm{~mm}$ and a thickness of $3 \mathrm{~mm}$. The dimensions of the sensor may be fairly large, but will be reduced in later designs to facilitate the integration of multiple sensors in resin braces, making the simultaneous multichannel measurement convenient for practical clinical applications.

\subsubsection{Force analysis}

Before analyzing the force situation of the sensor, let us look at the following two force scenarios.

Scenario 1: Assume there is a component with a typical cantilever beam structure, where one end is fixed and force is applied to the other end. ${ }^{(14)}$ The component deformation after force application is shown in Fig. 4, where $L$ is the thickness and $d$ is deformation. It can be seen from Fig. 4 that stretching in the front and contraction in the back of the component occurred at different degrees.

Scenario 2: Assume that equal shearing force acts on both ends of the component. Its deformation $d$ is shown in Fig. 5.

Now, suppose that there is an equal force on the silicone pad of the sensor. By force analysis, we can determine that this force is on the beam structure through the metal pad and the silicone pad. Figure 6 shows the ANSYS simulation diagram of the force on the beam structure.

For force condition analysis with ANSYS, the physical model of the beam is meshed and a three-dimensional finite mode is formed. ${ }^{(15)}$ For force $F$, the equivalent stress and equivalent strain echograms of the beam are shown in Figs. 7(a) and 7(b), respectively. From the echograms, we can see that the middle of the beam structure basically experiences no force, whereas the side sections experience the same force in opposite directions. 


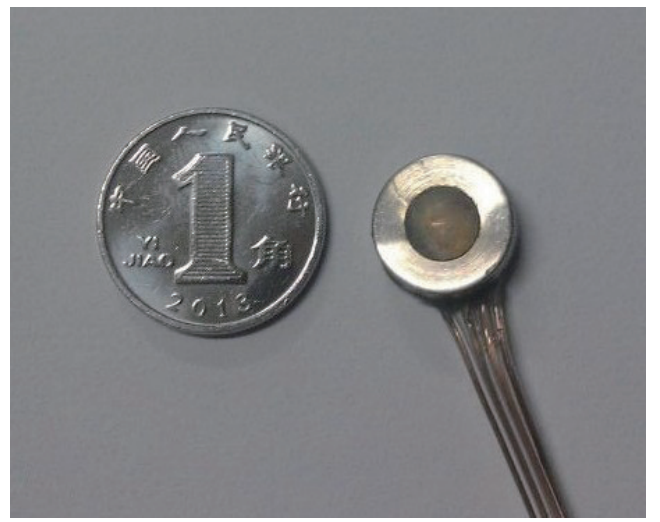

Fig. 3. (Color online) Appearance of sensor.

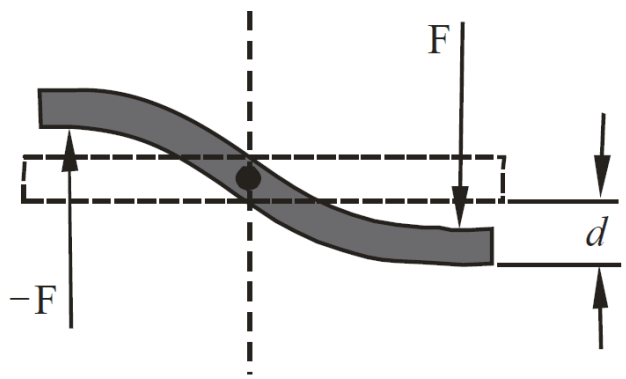

Fig. 5. Equal shearing force acts on both ends of component.

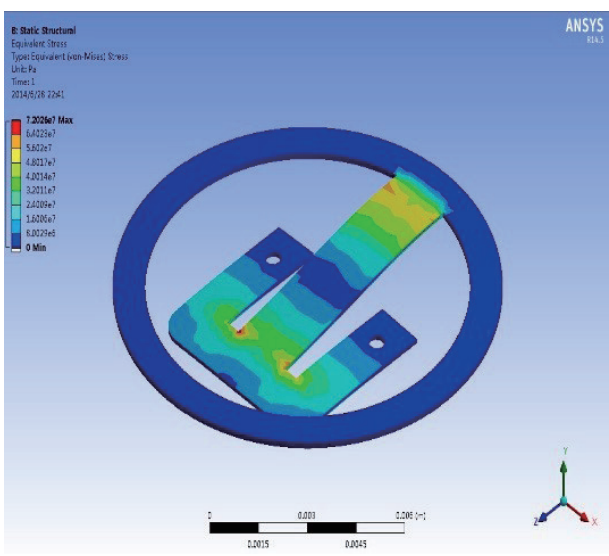

(a)

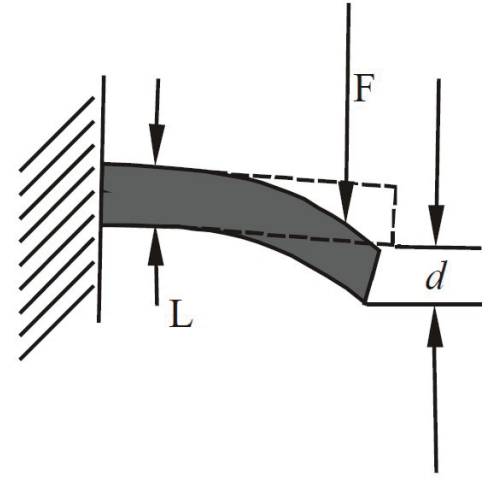

Fig. 4. Beam with one end fixed and with force deformation.

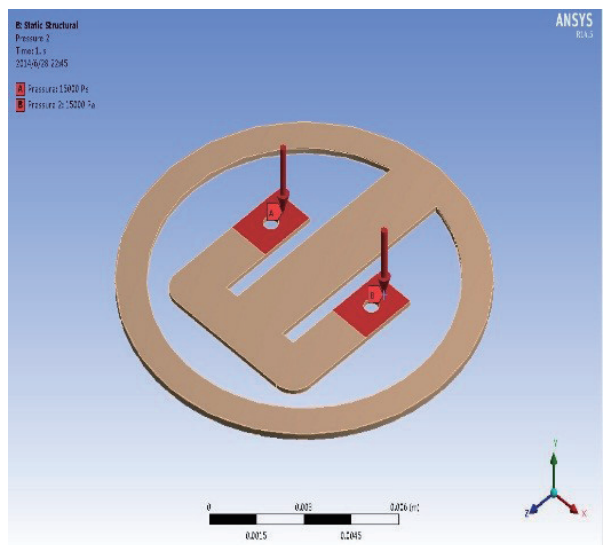

Fig. 6. (Color online) ANSYS simulation force on tablet beam.

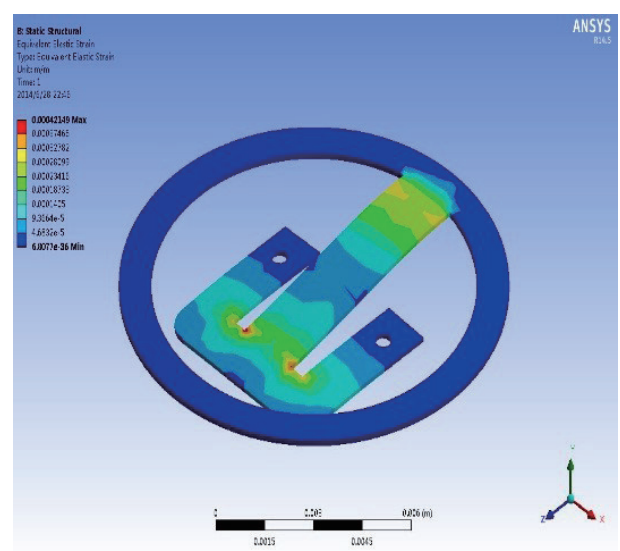

(b)

Fig. 7. (Color online) Equivalent stress and equivalent strain echograms. 
Judging from the results of the ANSYS analysis, the force situation of the component fits well with the second case of the previous analysis. On the basis of the situation of deformation, in the resistance strain gauge in Fig. 2, R1 stretched and R2 contracted, and their corresponding resistances also changed.

The internal structure of the resistance strain gauge is surrounded by many resistance wires, and its deformation structure is shown in Fig. 8, where dotted lines show the original shapes and solid lines show the deformed shapes. The length $\mathrm{L}$ of the sensitive grid of the resistance strain gauge used in the research is $1 \mathrm{~mm}$. Assuming the number of resistance wires is $\mathrm{n}$ and the change in the length of the resistance wire is $2 n \Delta L$, the change in the resistance is

$$
\Delta R=2 n f(\Delta L)
$$

$f$ denotes the relation function between resistance and the length $L$ of the sensitive grid of the resistance strain gauge.

\subsection{Circuit analysis}

From the working principle of the resistance strain gauge, we know that the change in the resistance is small $\left(\Delta R=10^{-1}-10^{-4} \Omega\right)$ because the mechanical strain range is very small, such that resistance cannot be measured directly using a conventional resistance measuring instrument. Therefore, we usually determine it from voltage signals, and then measure it using an electrical measurement instrument. In this study, we used a classical Wheatstone bridge circuit, the structure of which is shown in Fig. 9.

In Fig. 9, $U$ is the DC power voltage, $R_{1}$ and $R_{2}$ are the resistance strain gauges that are mounted on the stressed structure of the pressure sensor, and $R_{3}$ and $R_{4}$ are precision metal film resistors. As seen from the circuit diagram, the output voltage of the bridge $\left(U_{o}\right)$ is

$$
U_{o}=\frac{R_{1}}{R_{1}+R_{2}} U-\frac{R_{3}}{R_{3}+R_{4}} U=\frac{R_{1} R_{4}-R_{2} R_{3}}{\left(R_{1}+R_{2}\right)\left(R_{3}+R_{4}\right)} U
$$
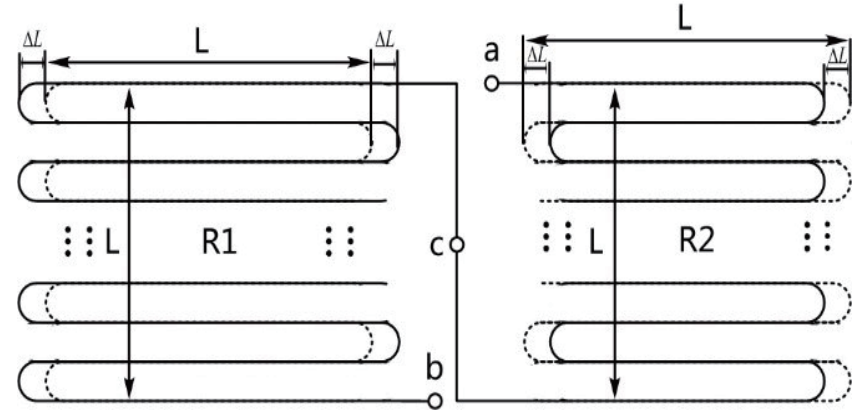

Fig. 8. Deformation structure of resistance strain gauge.

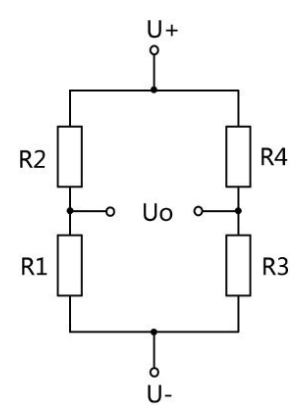

Fig. 9. Wheatstone bridge circuit. 
Suppose the bridge is balanced before the measurement, $R_{1} R_{4}=R_{2} R_{3}$, and the resistance strain gauges are placed as shown in Fig. 2. Then the resistance strain gauge $R_{1}$ will increase by $\Delta R_{1}$ and $R_{2}$ will decrease by $\Delta R_{2}$ in the measurement process.

$$
U_{o}=\frac{\Delta R_{1} R_{4}-\Delta R_{2} R_{3}}{\left(R_{1}+\Delta R_{1}+R_{2}+\Delta R_{2}\right)\left(R_{3}+R_{4}\right)} U
$$

The values of $\Delta R_{1}$ and $\Delta R_{2}$ are negligible; therefore,

$$
U_{o}=\frac{\Delta R_{1} R_{4}-\Delta R_{2} R_{3}}{\left(R_{1}+R_{2}\right)\left(R_{3}+R_{4}\right)} U
$$

In order to achieve the circuit balance where $R_{1} R_{4}=R_{2} R_{3}$ in the circuit design, we introduced a variable resistor of $10 \Omega$ in the circuit. The actual bridge circuit is shown in Fig. 10.

Because the output voltage $U_{o}$ of the circuit is very small, we used a precision differentialinput amplifier to enlarge the sensor signal. The whole circuit of the amplifier is shown in Fig. 11.

\subsection{Sensor manufacture}

The strain gauges chosen for this study are the high-precision strain gauges (BF120-1AA) produced by Hanzhong. They have a fully enclosed structure on a modified phenolic substrate. Constantan film is used as the sensitive grid material. The dimensions of the substrate are 4.2 $\times 2.8 \mathrm{~mm}^{2}$. The BF120-1AA strain gauges have the temperature and creep self-compensation functions. Their resistance is $120 \Omega$ and their sensitivity ratio is $2.10 \%$. The dimensions of the sensitive grid are $1.0 \times 1.7 \mathrm{~mm}^{2}$, and their accuracy grade is A. The BF120-1AA strain gauges are shown in Fig. 12.

To ensure that the strain gauges are firmly attached to the beam and to reduce residual stress, we must solidify the adhesive. Moreover, we must repeat the temperature circulating test and the alternating stress circulating test. ${ }^{(16)}$ The strain gauges after bonding were placed

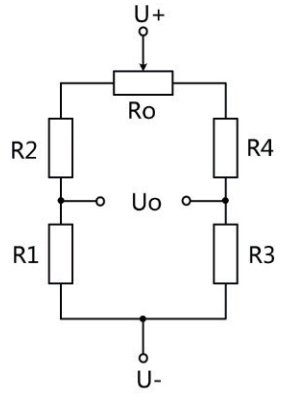

Fig. 10. Actual bridge circuit.

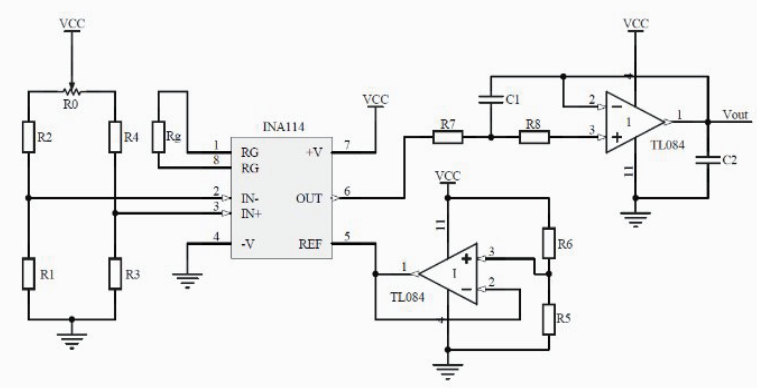

Fig. 11. Preamplifier circuit of the sensor. 


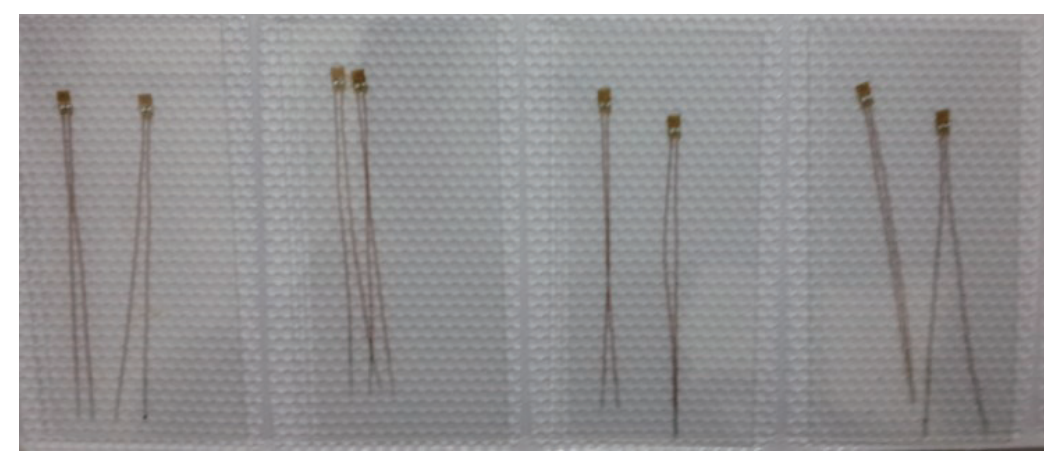

Fig. 12. (Color online) BF120-1AA strain gauges.

between two boards that were clamped and pressed by clips. The boards were placed into the heating box and heated at $120{ }^{\circ} \mathrm{C}$ for $45 \mathrm{~min}$. Then, under pressure, they were cooled to room temperature under ambient conditions. The boards were returned to the heating box, heated at $180{ }^{\circ} \mathrm{C}$ for $90 \mathrm{~min}$, and cooled to room temperature under ambient conditions after releasing pressure. Finally, the boards were again placed into the heating box, heated at $280{ }^{\circ} \mathrm{C}$ for 120 min, and cooled to room temperature in the heating box.

The beam with strain gauges pasted to both sides is shown in Fig. 13. After obtaining the elastic element, it must be protected by waterproofing because it will be used in the oral cavity. The elastic element is installed into the base with a waterproof layer, and then this unit is installed in the shell of the sensor. The sensor with the installed waterproofed elastic element in the shell is shown in Fig. 14.

Considering the oral environment, we use a soft sensitive element without sharp chamfering to ensure that the tested object will not be damaged during the process of testing. Thus, a domed resin, as shown in Fig. 15, was selected as the sensitive element of the sensor. The sensor was completely encapsulated after the sensitive element was installed (Fig. 16).

\section{Extreme Learning Machine}

The extreme learning machine (ELM) is a new algorithm of the single-hidden layer feedforward neural network (SLFN). ${ }^{(17)}$ This new algorithm can randomly generate the link weights of the input layer and hidden layer and the thresholds of hidden layer neurons. The weights and thresholds do not require adjustment in the process of training, and only the number of hidden layer neurons should be set to obtain the optimal solution. The algorithm has some advantages, such as its faster learning, good generalization performance in classification and regression problems, and wide use in the areas of classification and pattern recognition. ${ }^{(18-20)}$ In this study, the ELM was used to modify the nonlinear relationship between tongue pressure and voltage. 


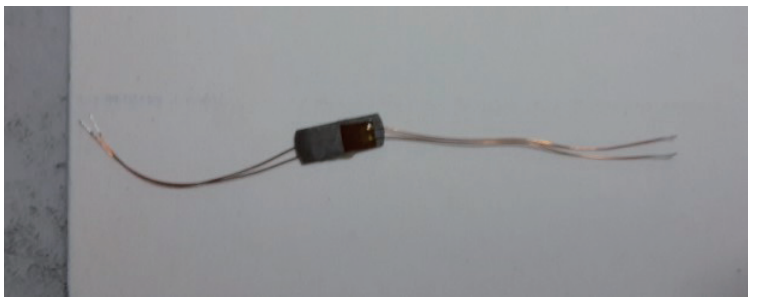

Fig. 13. (Color online) Beam with strain gauges.

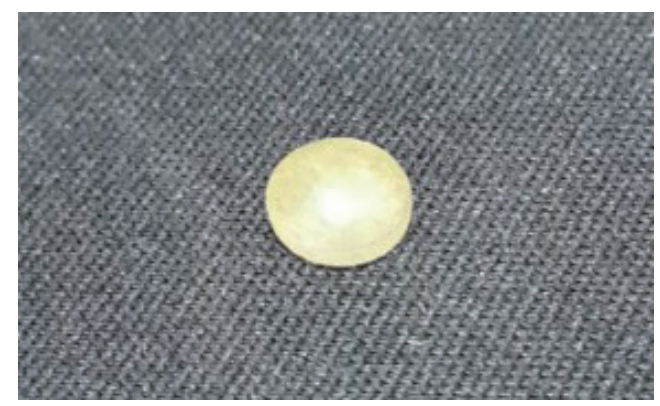

Fig. 15. (Color online) Domed resin.

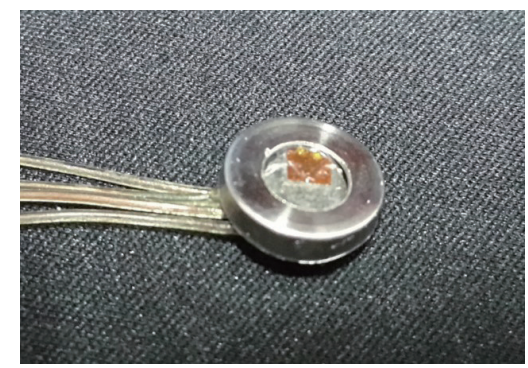

Fig. 14. (Color online) Sensor protected by waterproofing.

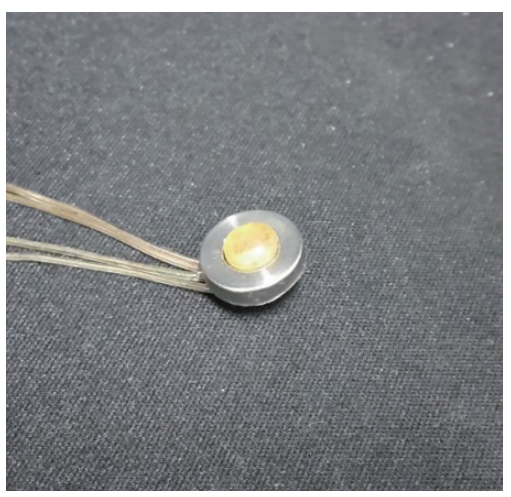

Fig. 16. (Color online) Sensor after encapsulation.

\subsection{SLFN}

The structure of a typical SLFN is shown in Fig. 17. It consists of an input layer, hidden layer, and output layer. The neurons are fully connected between the input and hidden layers, and between the hidden and output layers. The input layer has $n$ neurons corresponding to the $n$ input variables, the hidden layer has one neuron and the output layer has $m$ neurons correspond to the $m$ output variables. For consistency, the connective weights $\omega$ between the input and hidden layers are set to

$$
w=\left[\begin{array}{cccc}
\omega_{11} & \omega_{12} & \ldots & \omega_{1 n} \\
\omega_{21} & \omega_{22} & \ldots & \omega_{2 n} \\
\vdots & \vdots & & \vdots \\
\omega_{l 1} & \omega_{l 2} & \ldots & \omega_{l n}
\end{array}\right]_{l \times n}
$$

where $\omega_{i j}$ is the connective weight between the $i$ th neuron of the input layer and the $j$ th neuron of the hidden layer.

The connective weights $\beta$ between the hidden layer and the output layer are set to 


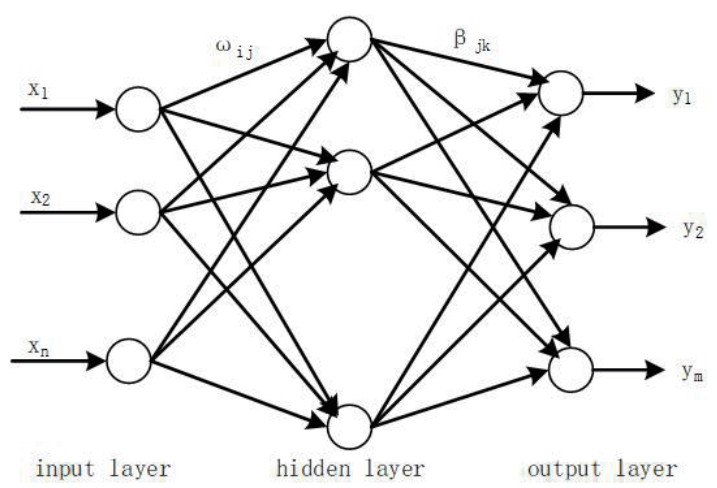

Fig. 17. Structure of typical SLFN.

$$
\beta=\left[\begin{array}{cccc}
\beta_{11} & \beta_{12} & \ldots & \beta_{1 m} \\
\beta_{21} & \beta_{22} & \ldots & \beta_{2 m} \\
\vdots & \vdots & \vdots & \vdots \\
\beta_{l 1} & \beta_{l 2} & \ldots & \beta_{l m}
\end{array}\right]_{l \times m}
$$

where $\beta_{j k}$ is the connective weight between the $j$ th neuron of the hidden layer and the $k$ th neuron of the output layer.

The thresholds $b$ of the hidden layer neuron are set to

$$
b=\left[\begin{array}{c}
b_{1} \\
b_{2} \\
\vdots \\
b_{l}
\end{array}\right]_{l \times m} .
$$

Suppose we have a training set with $Q$ samples, input matrix $X$, and output matrix $Y$. The activation function of the hidden layer neuron is set to $g(x)$. Then we can obtain the output $T$ of the network as shown in Fig. 17.

$$
\begin{gathered}
T=\left[t_{1}, t_{2}, \cdots, t_{Q}\right]_{m \times Q}, \\
t_{j}=\sum_{i=1}^{m} \beta_{i} g\left(w_{i} x_{j}+b_{i}\right)(j=1,2, \cdots, Q) .
\end{gathered}
$$

Here, $w_{i}=\left[w_{i 1}, w_{i 2}, \cdots, w_{m}\right], x_{j}=\left[x_{1 j}, x_{2 j}, \cdots, x_{n j}\right]^{\mathrm{T}}$.

The output of the network can also be represented as 


$$
H \beta=T^{\prime},
$$

where $T^{\prime}$ is the matrix transpose of matrix $T$ and $H$ is the output matrix of the hidden layer. The detailed description is

$$
\begin{gathered}
H\left(w_{1}, w_{2}, \cdots, w_{m}, b_{1}, b_{2}, \cdots b_{m}, x_{1}, x_{2}, \cdots, x_{Q}\right) \\
=\left[\begin{array}{ccccc}
g\left(w_{1} \cdot x_{1}+b_{1}\right) & \ldots & g\left(w_{2} \cdot x_{1}+b_{2}\right) & \ldots & g\left(w_{m} \cdot x_{1}+b_{m}\right) \\
g\left(w_{1} \cdot x_{2}+b_{1}\right) & \ldots & g\left(w_{2} \cdot x_{2}+b_{2}\right) & \ldots & g\left(w_{m} \cdot x_{2}+b_{m}\right) \\
\vdots & & \vdots & & \vdots \\
g\left(w_{1} \cdot x_{Q}+b_{1}\right) & \ldots & g\left(w_{2} \cdot x_{Q}+b_{2}\right) & \ldots & g\left(w_{m} \cdot x_{Q}+b_{m}\right)
\end{array}\right]_{Q \times m} .
\end{gathered}
$$

On the basis of Eq. (17), a new algorithm of ELM for the SLFN was proposed in Ref. 17.

\subsection{Principle of ELM}

For SLFNs, ELM can initialize input weights and bias in a random manner and obtain the corresponding output weights. On the basis of former studies, Huang et al. proposed two theorems.

Theorem 1: Given a standard SLFN with $N$ hidden nodes and the activation function $g$ : $R \rightarrow R$, which is infinitely differentiable in any interval, for $N$ arbitrary distinct samples $\left(x_{i}, t_{i}\right)$, where $x_{i} \in R^{n}$ and $t_{i} \in R^{m}$, for any $w_{i}$ and $b_{i}$ randomly chosen from any intervals of $R^{n}$ and $R$, respectively, in accordance with any continuous probability distribution when the probability is one, the hidden layer output matrix $H$ of the SLFN is invertible and $\|H \beta-T\|=0$.

Theorem 2: Given any small positive value 40 and the activation function $g: R \rightarrow R$, which is infinitely differentiable in any interval, there exists $\tilde{N} \leq N$ such that for $N$ arbitrary distinct samples $\left(x_{i}, t_{i}\right)$, where $x_{i} \in R^{n}$ and $t_{i} \in R^{m}$, for any $w_{i}$ and $b_{i}$ randomly chosen from any interval of $R^{n}$ and $R$, respectively, in accordance with any continuous probability distribution when the probability is one, $\left\|H_{N \times \tilde{N}} \beta_{\tilde{N} \times m}-T_{N \times m}\right\|<\varepsilon$.

The ELM can randomly generate the connective weights between the input and hidden layers and the thresholds $b$ of neurons in the hidden layer before training. Needing only knowledge of the number of neurons in the hidden layer and the activation function of a neuron in the hidden layer, we can determine the connective weights $\beta$ between the hidden layer and the output layer. The ELM algorithm mainly includes the following steps.

Step 1: Determine the number of neurons in the hidden layer, then randomly set the connective weights between the input and hidden layers and the thresholds $b$ of neurons in the hidden layer;

Step 2: Select a differentiable function as the activation function of neurons in the hidden layer, then compute the output matrix $H$ of the hidden layer;

Step 3: Compute the output layer weights $\beta^{\prime}: \beta^{\prime}=H^{+} T^{\prime}$. 


\section{Experiment}

For convenience in installing in patients, the sensor was embedded in medical composite resin and designed as a dental brace. The sensor can detect the change in pressure exerted by the tongue against the upper jaws during swallowing and chewing. The packaged pressure sensor in resin is shown in Fig. 18. In order to collect sensor data, the data acquisition unit is designed to have five collection channels and to carry out sampling continuously for $8 \mathrm{~h}$. The acquisition system has two modes: the sleep mode and swallowing mode. It can also detect the posture of sleeping patients, such as supine, left lateral decubitus, and right lateral. During the performance test, the sensor is pressed by the pressure gauge, and the pressure values are checked. The inspection instrument consists of a collector and a pressure gauge, as shown in Fig. 19.

In the process of verification, the formula for tongue pressure is

$$
P=\frac{F}{S},
$$

where $P$ is the pressure, $F$ is the tongue pressure, and $S$ is the force-bearing area between the silicone pad and the tongue, which is about $0.0003 \mathrm{~m}^{2}$.

To clarify the relationship between the output voltage and tongue pressure, the pressure applied to the sensor was calibrated with a calibrating instrument, as shown in Table 1.

We checked the sensor thirteen times, then used the mean for nonlinear compensation. The results of checking are shown in Table 2. The plots of these results are shown in Fig. 20.

The least-squares method (LSM) is very effective for curve fitting. Although simple, it can basically satisfy different applications. The curve of the relationship between output voltage and tongue pressure obtained by the LSM is shown in Fig. 21. The mathematical relationship between voltage $v$ and tongue pressure $P$ is

$$
P=3.19 v^{2}+1.907 v-6.919 .
$$

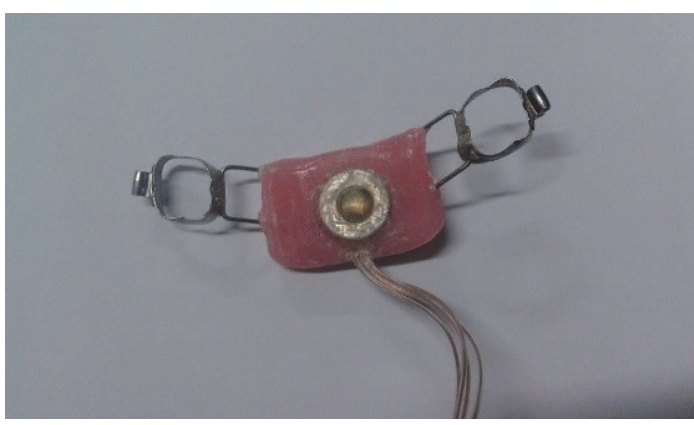

Fig. 18. (Color online) Packaged pressure sensor in resin.

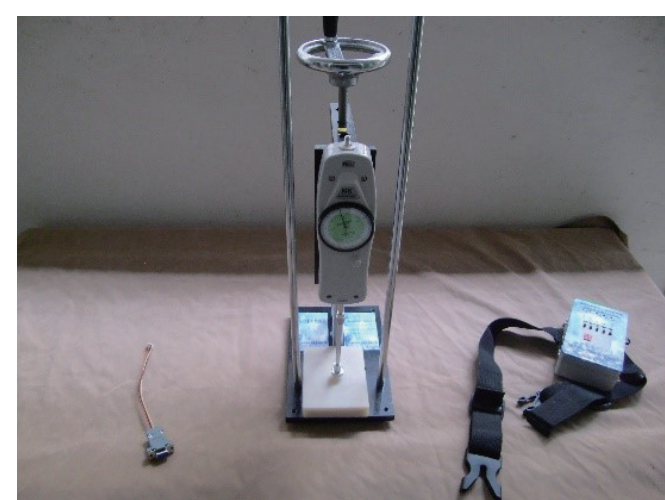

Fig. 19. (Color online) Inspection instrument. 
Table 1

Force and tongue pressure.

\begin{tabular}{lc}
\hline$P(\mathrm{kPa})$ & $F(\mathrm{~N})$ \\
\hline 0 & 0.00 \\
2.5 & 0.75 \\
5 & 1.50 \\
7.5 & 2.25 \\
10 & 3.00 \\
12.5 & 3.75 \\
15 & 4.50 \\
17.5 & 5.25 \\
20 & 6.00 \\
22.5 & 6.75 \\
\hline
\end{tabular}

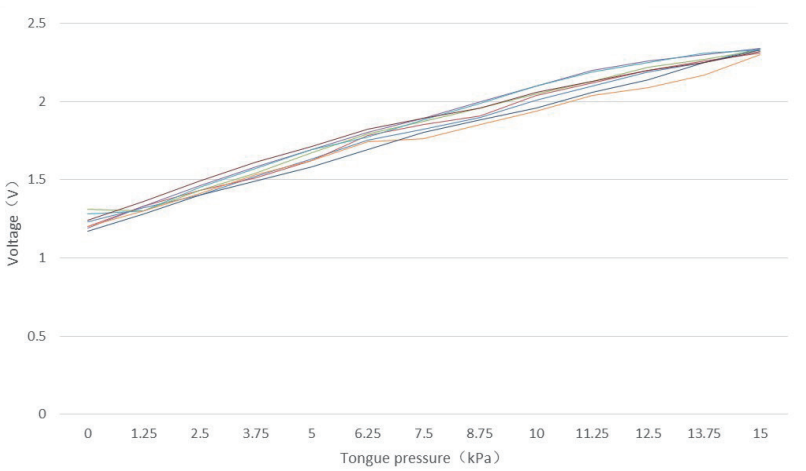

Fig. 20. (Color online) Plots of the calibration result.

Table 2

Result of calibration.

\begin{tabular}{cccccccccc}
\hline$P(\mathrm{kPa})$ & $\mathrm{U} 1(\mathrm{~V})$ & $\mathrm{U} 2(\mathrm{~V})$ & $\mathrm{U} 3(\mathrm{~V})$ & $\mathrm{U} 4(\mathrm{~V})$ & $\mathrm{U} 5(\mathrm{~V})$ & $\mathrm{U} 6(\mathrm{~V})$ & $\mathrm{U} 7(\mathrm{~V})$ & U8 $(\mathrm{V})$ & Average \\
\hline 0.00 & 1.23 & 1.20 & 1.31 & 1.19 & 1.28 & 1.20 & 1.17 & 1.24 & 1.23 \\
1.25 & 1.32 & 1.33 & 1.30 & 1.33 & 1.30 & 1.30 & 1.28 & 1.36 & 1.32 \\
2.50 & 1.40 & 1.43 & 1.43 & 1.46 & 1.45 & 1.41 & 1.40 & 1.49 & 1.43 \\
3.75 & 1.52 & 1.51 & 1.54 & 1.58 & 1.57 & 1.53 & 1.49 & 1.61 & 1.54 \\
5.00 & 1.63 & 1.62 & 1.67 & 1.69 & 1.69 & 1.62 & 1.58 & 1.71 & 1.65 \\
6.25 & 1.75 & 1.78 & 1.79 & 1.80 & 1.77 & 1.74 & 1.69 & 1.82 & 1.76 \\
7.50 & 1.82 & 1.85 & 1.87 & 1.89 & 1.88 & 1.76 & 1.8 & 1.89 & 1.85 \\
8.75 & 1.90 & 1.91 & 1.96 & 2.00 & 1.99 & 1.85 & 1.88 & 1.96 & 1.93 \\
10.00 & 2.01 & 2.04 & 2.05 & 2.10 & 2.10 & 1.94 & 1.96 & 2.06 & 2.03 \\
11.25 & 2.10 & 2.12 & 2.13 & 2.20 & 2.19 & 2.04 & 2.06 & 2.13 & 2.12 \\
12.50 & 2.19 & 2.20 & 2.22 & 2.26 & 2.25 & 2.09 & 2.14 & 2.20 & 2.19 \\
13.75 & 2.25 & 2.26 & 2.27 & 2.30 & 2.31 & 2.17 & 2.25 & 2.25 & 2.26 \\
15.00 & 2.34 & 2.31 & 2.33 & 2.34 & 2.33 & 2.30 & 2.33 & 2.32 & 2.33 \\
\hline
\end{tabular}

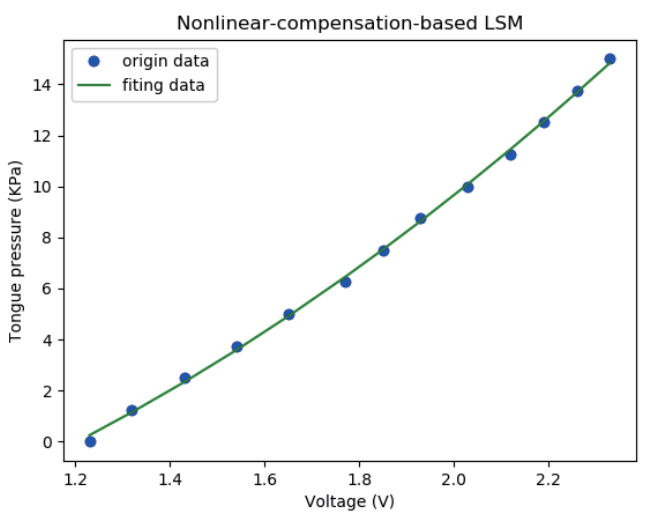

Fig. 21. (Color online) Curve of least-squares fitting.

If there is much data or data is complicated, the LSM is highly disadvantageous. Therefore, we optimized the curve using the ELM, which has a higher learning rate and good generalization performance. 
Following the steps above, we also used the data in Table 2 to modify the nonlinear relationship between voltage and tongue pressure with the ELM. Because the connective weights between the input and hidden layers and the thresholds $b$ of neurons in the hidden layer were selected randomly, there were differences among the results of all runs. We selected the best-fit results, as shown in Fig. 22.

As can be seen in Fig. 22, the nonlinear compensation model based on ELM is clearly better than the nonlinear compensation model based on LSM. In addition, we selected other 11 calibration data to experiment and compare the measuring accuracy of the above-mentioned two nonlinear compensation models, and the measurement results as shown in Table 3.

Linearity is an important indicator of the static nature of the sensor, and shows the proximity of the fitting curve to the characteristic curve. ${ }^{(21)}$ It is often expressed as the ratio of the maximum deviation between the characteristic curve and the fitted curve to the full-span output. The smaller the value, the better the linear characteristics of the sensor. The equation is

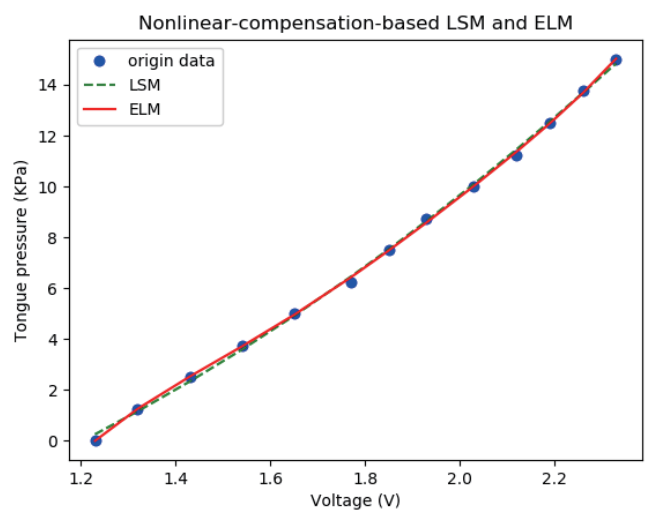

Fig. 22. (Color online) Nonlinear compensation model based on ELM.

Table 3

Measurement results for LSM model and ELM model.

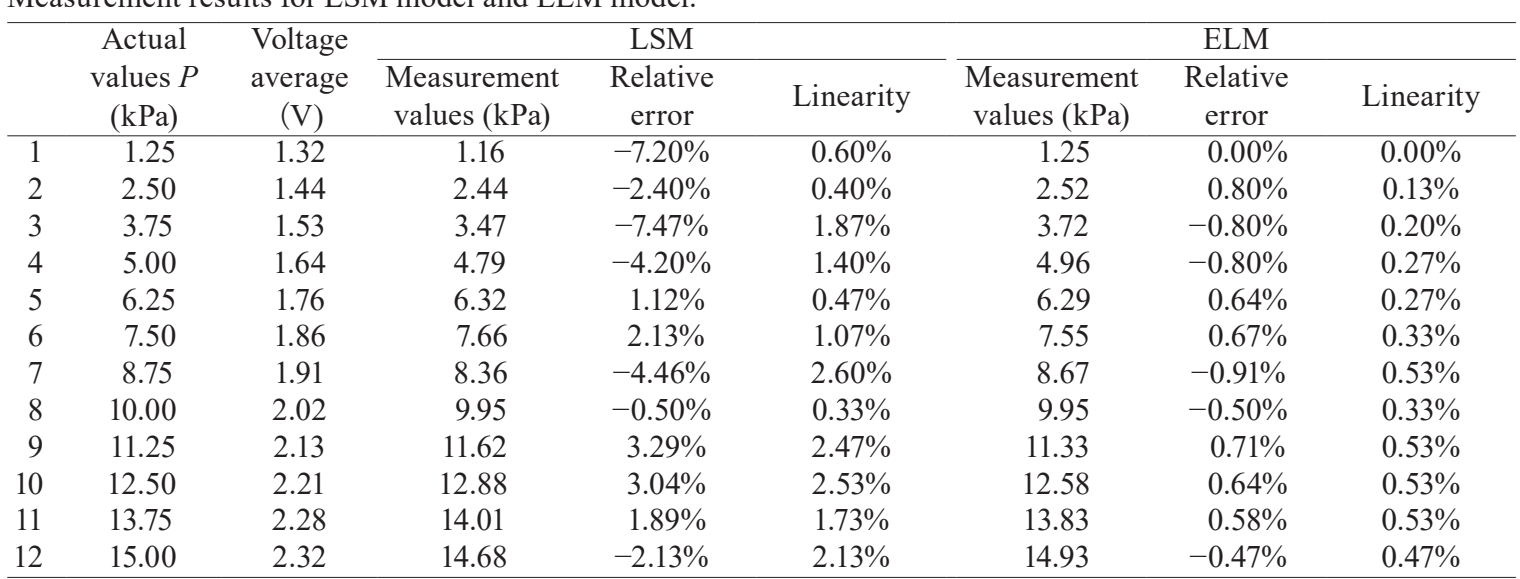

Note: relative error $=($ measurement value - actual value $) /$ actual value $\times 100 \%$ 


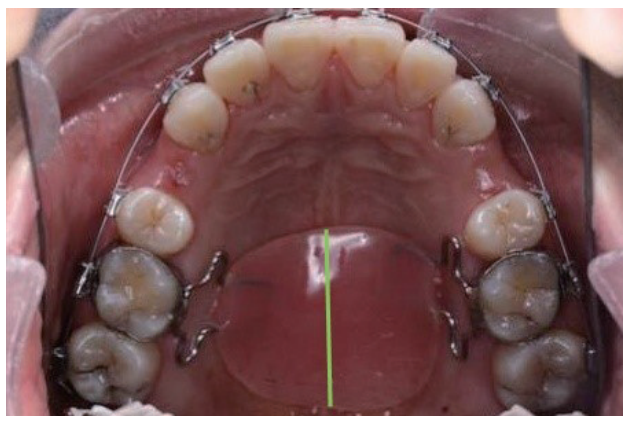

Fig. 23. (Color online) Install location of sensor.

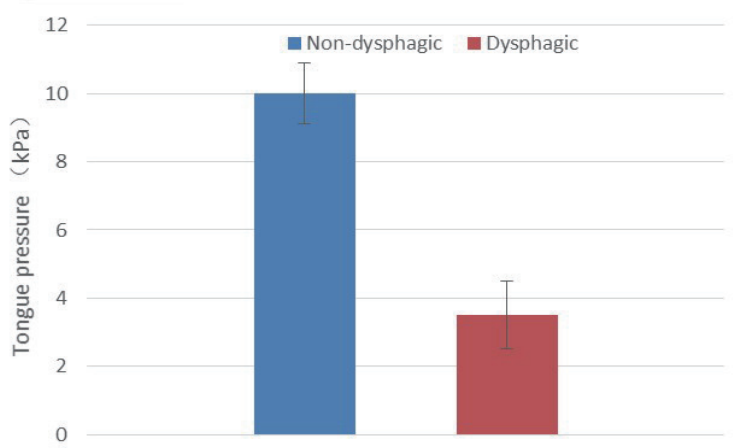

Fig. 24. (Color online) Maximal tongue pressure in nondysphagia group and dysphagia group.

$$
\xi_{L}= \pm \frac{\Delta L_{\max }}{Y_{f s}} \times 100 \%
$$

where $\Delta L_{\max }$ is the maximum deviation between the mean calibration curves and the fitted lines and $Y_{f s}$ is the theoretical full-span output.

From Table 3, we can see that the linearity of the designed sensor based on the ELM is less than that of a sensor based on LSM, which confirms that the linear characteristics of the designed sensor based on the ELM is better. Table 3 also shows that the measurement precision of the sensor based on the ELM can achieve $0.91 \%$, indicating that the designed sensor has good precision and reliability.

After fabricating the designed sensor, we installed it at the center of the oral palate, as shown in Fig. 23. Then, the tongue pressure test was carried out.

Twenty dysphagia patients and 20 healthy volunteers for comparison were recruited as subjects of this study. The 20 dysphagia patients (10 males, 10 females) had an average age of 60 and the 20 healthy volunteers ( 8 males, 12 females) had an average age of 65 . All the subjects sat in a chair with their feet put on the ground. Each swallowed $3 \mathrm{ml}$ of water while keeping the head position and performed the test three times. Figure 24 shows the maximal tongue pressures in nondysphagia group and dysphagia group. It can be seen from Fig. 24 that the maximal tongue pressure was significantly higher in the nondysphagia group $(10 \pm 0.9 \mathrm{kPa})$ than in the dysphagia group $(3.5 \pm 1 \mathrm{kPa})$.

\section{Conclusions}

We designed a sensor for measuring tongue pressure to assist doctors in diagnosing dysphagia. Regarding the problem of nonlinearity between the tongue pressure and the voltage, the ELM is used. Experiments prove that this method has better linearity, higher precision, smaller error, and other advantages than the LSM. However, the repeatability and accuracy of the designed sensor based on ELM are not satisfactory, because of many factors, including structure, materials, and circuit of the designed sensor. Further improvement is needed. 


\section{References}

1 D. J. Ramsey, D. G. Smithard, and L. Kalra: Stroke 34 (2003) 1252.

2 R. Martino, N. Foley, S. Bhogal, N. Diamant, M. Speechley, and R. Teasell: Stroke 36 (2005) 2756.

3 N. A. Leopold and M. C. Kagel: Arch. Phys. Med. Rehabil. 64 (1983) 71.

4 Y. Utanohara, R. Hayashi, M. Yoshikawa, M. Yoshida, K. Tsuga, and Y. Akagawa: Dysphagia 23 (2008) 286.

5 S. Kumar, M. H. Selim, and L. R. Caplan: Lancet Neurol. 9 (2010) 105.

6 S. Wang, Y. Feng, Y. Chen, R. Zhang, and Y. Huang: Chin. J. Electron. 25 (2016) 77.

7 E. Brogan, C. Langdon, K. Brookes, C Budgeon, and D Blacker: Dysphagia 29 (2014) 340.

8 T. Ono, K. Hori, and T. Nokubi: Dysphagia 19 (2004) 259.

9 K. Hori, T. Ono, and T. Nokubi: J. Dent. Res. 85 (2006) 187.

10 J. Kieser, B. Singh, M. Swain, I. Ichim, J. N. Waddell, D. Kennedy, K. Foster, and V Livingstone: Dysphagia 23 (2008) 237.

11 K. Hori, T. Ono, H. Iwata, T. Nokubi, and I. Kumakura: Gerodontology 22 (2005) 227.

12 K. Hori, T. Ono, K. Tamine, J. Kondo, S. Hamanaka, Y Maeda, J. Dong, and M. Hatsuda: J. Prosthodont. Res. 53 (2009) 28.

13 Y. Li: Strain Pressure Institutions of Micro-sensor Integration of Electronic Manometers: Design of Pressure Sensor for Electronic Manometry (North University of China, Shanxi, 2009) p. 26.

14 J. Tang: The Study of a Novel Sensing Technology Based on Piezoelectric Microcantilever: Design of Sensor System of Piezoelectric Microcantilever (Tianjin University, Tianjin, 2005) p. 20.

15 F. Zhou and G. X. Wang: Group Technol. Prod. Modernization 28 (2011) 9.

16 F. Y. Yin and C. L. Wang: Weighing Instrum. 33 (2004) 13.

17 G. B. Huang, Q. Y. Zhu, and C. K. Siew: Neurocomputing 70 (2006) 489.

18 K. C. Sivalingam, S. Mahendran, and S. Natarajan: Int. J. Comput. Control 11 (2016) 372.

19 S. Kariminia, S. Shamshirband, S. Motamedi, R. Hashim, and C. Roy: Renewable Sustainable Energy Rev. 58 (2016) 751.

20 G. B. Huang, H. Zhou, X. Ding, and R. Zhang: IEEE Trans. Syst. Man Cybern. Part B 42 (2012) 513.

21 W. K. Shi and X. F. Yu: Measurement Technique: Test System (China Machine Press, Beijing, 2010) p. 29. 